\title{
Electroacupuncture promotes neural stem cell proliferation and neurogenesis in the dentate gyrus of rats following stroke via upregulation of Notch1 expression
}

\author{
JUNHONG ZHAO $^{1,2}$, MINGHONG SUI ${ }^{1}$, XIAO LÜ ${ }^{1}$, DONGMEI JIN ${ }^{1}$, ZHIQIANG ZHUANG $^{1}$ and TIEBIN YAN $^{1}$ \\ ${ }^{1}$ Department of Rehabilitation Medicine, Sun Yat-sen Memorial Hospital, Sun Yat-sen University, Guangzhou, Guangdong 510120; \\ ${ }^{2}$ Department of Rehabilitation Medicine, Second Affiliated Hospital of Guangzhou Medical University, \\ Guangzhou, Guangdong 510260, P.R. China
}

Received April 22, 2014; Accepted January 9, 2015

DOI: $10.3892 / \mathrm{mmr} .2015 .4279$

\begin{abstract}
Neural stem cells (NSCs) are important in rehabilitation following stroke. Electroacupuncture (EA) treatment has been observed to promote the recovery of neurological functions subsequent to stroke, however, the effects of EA on the proliferation and differentiation of NSCs and its potential mechanisms remain to be elucidated. In the present study, rats, in which a stroke was induced through middle cerebral artery occlusion (MCAO), were treated with EA or control manipulation for 21 days. The modified Neurological Severity score and Morris water maze tests were used to assess the neurological functions of the rats. Bromodeoxyuridine (BrdU)/glial fibrillary acidic protein (GFAP) or BrdU/neuronal marker (NeuN) double immunofluorescence staining were used to examine the proliferation and differentiation of the NSCs. Reverse transcription quantitative polymerase chain reaction (RT-qPCR) and western blot analyses were performed to detect the expression levels of Notch1 and Hes1 in the dentate gyrus (DG) of the hippocampus of rats following MCAO. The results demonstrated that EA treatment significantly improved the neurological functional recovery of rats following stroke. A significant increase was observed in the number of $\mathrm{BrdU}^{+} / \mathrm{GAFP}^{+}$and $\mathrm{BrdU}^{+} / \mathrm{NeuN}^{+}$cells in the $\mathrm{DG}$ area in the EA-treated rats compared with that of the control group. RT-qPCR analysis revealed that EA treatment significantly increased the expression levels of Notch1 and Hes1, which may account for the enhanced proliferation and differentiation of NSCs. In conclusion, to the best of our knowledge, the present study was the first to demonstrate that EA treatment promoted
\end{abstract}

Correspondence to: Professor Tiebin Yan, Department of Rehabilitation Medicine, Sun Yat-sen Memorial Hospital, Sun Yat-sen University, 107 West Yanjiang Road, Guangzhou, Guangdong 510120, P.R. China

E-mail: yantiebin11@163.com

Key words: stroke, electroacupuncture, neural stem cells, Notch1, dentate gyrus
NSC proliferation and neurogenesis in the DG area through the upregulation of Notch signaling following a stroke; therefore, EA may be a useful novel therapeutic strategy in future stroke treatment.

\section{Introduction}

Cerebrovascular diseases are one of the prominent causes of mortality and permanent disability worldwide, the occurrence of which is predicted to further increase due to increasing life expectancies (1). The pharmaceutical industry has developed numerous potential neuroprotective drugs for the treatment of stroke, which have been observed to decrease cerebral damage and enhance the recovery of motor and cognitive abilities following ischemic insult in preclinical studies using animal models. However, these results have not been reproduced in phase III clinical trials and none of the assessed compounds led to the successful post-ischemic treatment of patients $(2,3)$. Therefore, further investigation in this field is required in order to examine novel therapeutic strategies, which may ameliorate the recovery of post-ischemic patients.

Stem cells, with self-renewal and pluripotent differentiation potentials, are a promising source for tissue regeneration, including neural regeneration (4). Neural stem cells (NSCs) have been identified in the subventricular zone (SVZ) and dentate gyrus (DG) of adult brain and these NSCs proliferate and generate neurons throughout the adult life (5-9). It is now generally considered that cerebral ischemia results in the expansion of endogenous NSCs, as has been previously demonstrated using various ischemia models, including transient global ischemia $(10)$, transient focal ischemia $(11,12)$ and permanent focal ischemia (13). Stroke, induced by middle cerebral artery occlusion (MCAO), leads to the increased proliferation of NSCs in SVZ and the formation of neuroblasts, which migrate towards the damaged striatum, where they differentiate into mature striatal neurons (12). In addition to replacing lost neurons, NSCs appear to contribute to regeneration following stroke by providing neuroprotection and trophic support, which reduces neuroinflammation and induces remodeling $(14,15)$. However, the spontaneous proliferation and differentiation of endogenous NSCs stimulated by stroke is often insufficient to 
overcome the neural damage (14). Therefore, it is important to develop novel strategies to enhance neurogenesis in order to improve rehabilitation following stroke.

Acupuncture has been used in traditional Chinese medicine for $>3,000$ years as a treatment for numerous diseases, including stroke. The use of acupuncture has become more prevalent in Western countries, and has been suggested to have potential therapeutic benefits for the treatment of cerebral ischemia-associated disorders $(16,17)$. A previous meta-analysis confirmed that acupuncture may be effective in the treatment of post stroke rehabilitation (18). Electroacupuncture (EA) is a form of acupuncture which involves a small electric current between pairs of acupuncture needles. EA is considered to augment the use of regular acupuncture (19) and several clinical trials have demonstrated that EA treatment improves limb function following stroke $(20,21)$. Studies involving murine models have demonstrated that EA treatment promotes neurological functional recovery through a series of mechanisms (22). Ke et al (23) reported that EA treatment upregulates the expression of brain-derived neurotrophic factor (BDNF), an important peptide, which supports the growth and maintenance of brain neurogenesis and facilitates motor recovery following stroke (23). However, whether EA treatment promotes neurogenesis through stimulating the proliferation of NSCs remains to be elucidated.

The present study aimed to examine the effects of EA treatment on the proliferation and differentiation of NSCs in the DG area of the adult rat brain following stroke, and to assess the impact of EA treatment on the expression of Notch1, an important molecule maintaining the proliferation of stem cells.

\section{Materials and methods}

Animal care. The experimental procedure used in the present study was approved by the Ethics Committee for Animal Experimentation of Sun Yat-sen University (Guangzhou, China) and was performed according to the Guidelines for Animal Experimentation of Sun Yat-sen University. All efforts were made to minimize animal suffering and the number of animals used. Male specific pathogen-free (SPF) Sprague-Dawley rats $(n=160)$, weighing 310 $\pm 30 \mathrm{~g}$, were provided by the Experimental Animal Center of Sun Yat-sen University and housed under controlled conditions with a 12 hour light/dark cycle, temperature of $24 \pm 1^{\circ} \mathrm{C}$ and humidity of $50 \pm 5 \%$ for at least 1 week prior to drug treatment or surgery. The rats were allowed free access to a standard rodent diet and tap water.

Experimental stroke in rats. The rats were anesthetized with an intraperitoneal injection of sodium pentobarbital (3\%; Sigma-Aldrich, St. Louis, MO, USA) at a dose of $30 \mathrm{mg} / \mathrm{kg}$. Core body temperature was monitored using a rectal probe (Zhengzhou Haorunqi Electronic Sci-Tech Co., Ltd., Henan, China) and were maintained at $37 \pm 0.5^{\circ} \mathrm{C}$ using a heating lamp and a heating pad (Zhengzhou Haorunqi Electronic Sci-Tech Co., Ltd.). The arterial blood gases, $\mathrm{pH}, \mathrm{PaO}_{2}, \mathrm{PaCO}_{2}$ and blood pressure were closely monitored via catheterizing the right femoral artery using a RM-6240BD physiological minitoring system (Chengdu Instrument Factory, Sichuan, China). MCAO was achieved using the Intraluminal Filament method, as previously described (24). Following exposure of the external carotid artery, the internal carotid artery (ICA) and the pterygopalatine artery of the ICA, a piece of monofilament nylon suture (diameter, $280 \mu \mathrm{m}$; Shadong Biotek, Beijing, China), with its tip rounded by gentle heating (diameter, $380 \pm 20 \mu \mathrm{m}$ ), was introduced via the lumen of the left external carotid artery stump and left ICA to embed into the left anterior cerebral artery, resulting in the occlusion of the right middle cerebral artery at its origin. Following surgery, the rats were transferred to their cage, in which the temperature was maintained at $37^{\circ} \mathrm{C}$ until the animals were completely conscious. Sham-operated rats (S group) were manipulated in the same way, however the ICA was not occluded.

Measurement of neurological deficits. Subsequent to the regaining consciousness, neurological deficits were preliminarily determined using a modified Bederson's scoring system $(25,26)$ as follows: 0 , no observable deficit; 1 , forelimb flexion; 2 , forelimb flexion with decreased resistance to lateral push; 3, unidirectional circling; 4, unidirectional circling with decreased level of consciousness. Rats with a score of 2-3 were selected for use in the subsequent experiments.

Electroacupuncture treatment. A total of 128 eligible rats with successful MCAO were randomly assigned into either the electroacupuncture treatment group (EA group) or control group ( $\mathrm{M}$ group). In the EA group rats, stainless acupuncture needles (diameter of $0.3 \mathrm{~mm}$ ) were inserted into the acupuncture points Baihui (DU20) and Shuigou (DU26) at a depth of 2-3 mm. Stimulation was then generated by the EA apparatus (Model G6805; SMIF, Shanghai, China), and the stimulation parameters were set as follows: Disperse wave, 4 and $20 \mathrm{~Hz}$; electric current, 1-2 mA; voltage, 2-4 mV; $15 \mathrm{~min}$ of each treatment; once a day. EA treatment was performed at $72 \mathrm{~h}$ following surgery and continued until the animals were sacrificed for tissue preparation. The rats in the $\mathrm{M}$ group were subjected to the same manipulation procedure without any electric stimulation.

Behavioral assessment. Behavioral assessments of the rats in the two groups were performed by an investigator in a blinded-manner. The assessments consisted of the modified Neurological Severity Score (mNSS) and Morris water maze. All measurements were completed in a dedicated behavioral investigation facility during this interval in order to minimize the environmental impact associated with transfer between home cage and the test arenas.

The mNSS test was performed at 3, 7, 14 and 21 days of EA or control treatment. Table I shows the mNSS scores (27). Neurological function was graded on a scale of 0 to 18 , with 0 indicating a normal score and 18 indicating the maximal deficit score. The mNSS is a composite of motor, sensory, reflex and balance tests. In the severity scores of injury, 1 score point is awarded for the inability to perform the test or for the lack of a reflex; thus, the higher the score, the more severe the injury (27).

The Morris water maze test was used to measure spatial learning and memory at days 7, 14 and 21 EA or control treatment, as previously described (28). Each rat was placed 
individually in a black water tank $(136 \mathrm{~cm}$ in diameter and $60 \mathrm{~cm}$ in height) in a well-lit room and filled with water $\left(50 \mathrm{~cm}\right.$ height at $27^{\circ} \mathrm{C}$ ). The tank was visually separated into four quadrants and, in the center of one quadrant, a plexiglass platform (diameter, $10 \mathrm{~cm}$; Zhengzhou Haorunqi Electronic Sci-Tech Co., Ltd.) was hidden $2 \mathrm{~cm}$ below the waterline. Numerous extra-maze cues surrounding the maze were fixed at specific locations and were visible to the rats. Following a single habituation trial, the rats were trained over 4 days and received four trials each day. On each trial, the rat had $120 \mathrm{sec}$ to escape to the submerged platform; rats that failed to escape were led to the platform and remained there for $30 \mathrm{sec}$ prior to being removed from the maze and dried off. On the fifth day, a probe trial was performed following removal of the platform. The latency of quadrant search, path length and swim speed were measured by a TSE video tracking system (TSE Systems, Bad Homburg, Germany) interfaced to a computer on days 3, 7,14 and 21 of treatment.

Immunofluorescence confocal microscopy. In order to track the fate of proliferating cells, the rats were administered with intraperitoneal (i.p.) injections of $75 \mathrm{mg} / \mathrm{kg}$ body weight bromodeoxyuridine (BrdU; Sigma-Aldrich) dissolved in phosphate-buffered saline (PBS; Whiga Technology Co., Ltd., Guangzhou, Guangdong, China) twice a day for 5 days prior to sacrifice. At days 3, 7, 14 and 21 of EA or control treatment, rats ( $n=5$ per experiment subgroup per time-point) were sacrificed through deep anesthetization with $45 \mathrm{mg} / \mathrm{kg}$ sodium pentobarbital and perfused intracardially with cold PBS and $4 \%$ buffered paraformaldehyde. Brains were paraffin-embedded (Whiga Technology Co., Ltd.) and sliced into 5- $\mu \mathrm{m}$ thick coronal sections, starting at $3 \mathrm{~mm}$ posterior to the anterior pole. The sections were then incubated with the mixture of monoclonal mouse antibodies (1:400) for BrdU (cat. no. MCA2060T; Oxford Biotech, Oxford, UK) with polyclonal mouse antibodies (1:400) for neuronal nuclei (NeuN; cat. no. SAB4300883; Sigma-Aldrich) or polyclonal mouse antibodies $(1: 10,000)$ for glial fibrillary acidic protein (GFAP; cat. no. SAB1405864; Sigma-Aldrich) overnight at $4{ }^{\circ} \mathrm{C}$, followed by a mixture of fluorescein isothiocyanate-conjugated goat anti-rabbit immunoglobulin (Ig)G and cyanine dye (Cy)3-conjugated goat anti-mouse IgG (1:100; Jackson Laboratory, Bar Harbor, ME, USA). The sections were washed in Tris-buffered saline (TBS; $0.2 \%$ Triton X-100 in PBS; Whiga Technology Co., Ltd.) and mounted with Vectashield mounting medium (Vector Laboratories, Burlingame, CA, USA). Confocal images were captured using an LSM710 confocal spectral microscope (magnification, x400; ZEISS, Oberkochen, Germany). Image analysis was performed using Leica QWin software (Leica Microsystems, Weltzlar, Germany). The number of cells showing double immunostaining was estimated by counting cells in five random fields of the peri-necrotic cortex in five non-continuous sections.

Reverse transcription quantitative polymerase chain reaction (RT-qPCR). Following evaluation of neurological deficits, rats ( $n=6$ per experiment group per time-point) were sacrificed with an overdose of sodium pentobarbital at days 3, 7, 14 and 21 of EA or control treatment, as described above. Total RNA was extracted from the perinecrotic cortex using TRIzol reagent (Invitrogen Life Technologies, Carlsbad, CA, USA) according to the manufacturer's instructions. RNA $(2 \mu \mathrm{g})$ was then reverse transcribed into first-strand cDNA using M-MLV Reverse Transcriptase (Promega Corp., Madison, WI, USA) according to the manufacturer's instructions. Notch1, Hes1 and GAPDH were amplified by qPCR using the following primers: Notch1, forward 5'-TCGTGCTCCTGTTCTTTGTG-3' and reverse 5'-GGGTTCTCTCCGCTTCTTCT-3'; Hes1, forward 5'-GCTGGAGAAGGCAGACATTC-3' and reverse 5'-GTCACCTCGTTCATGCACTC-3'; and GAPDH, forward 5'-TGCCACTCAGAAGACTGTGG-3' and reverse, 5'-TTCAGCTCTGGGATGACCTT-3' (BGI Tech, Guangdong, China). Gene-specific amplification was performed in an ABI 7500 real-time PCR system (Life Technologies, Carlsbad, CA, USA) using $15 \mu \mathrm{l}$ PCR mix containing $0.5 \mu \mathrm{l}$ cDNA, $7.5 \mu 12 \mathrm{x}$ SYBR Green master mix (Invitrogen Life Technologies) and $200 \mathrm{nM}$ of the appropriate primers. The mix was preheated at $95^{\circ} \mathrm{C}$ for $30 \mathrm{sec}$ and amplified in 45 cycles of $95^{\circ} \mathrm{C}$ for $5 \mathrm{sec}$ and $60^{\circ} \mathrm{C}$ for $34 \mathrm{sec}$. The resolution curve was measured at $95^{\circ} \mathrm{C}$ for $15 \mathrm{sec}, 60^{\circ} \mathrm{C}$ for $15 \mathrm{sec}$ and $95^{\circ} \mathrm{C}$ for $15 \mathrm{sec}$. The threshold cycle $(\mathrm{Ct})$ value of each sample was calculated and the relative expression of Notch1 and Hes1 mRNA was normalized to the GAPDH value (2- ${ }^{-\mathrm{Ct}}$ method) (29).

Statistical analysis. All values are expressed as mean \pm standard deviation. A repeated measurement analysis of variance was performed to analyze differences in the behavioral assessment data and relative mRNA expression levels. Analysis of variance and the Least Significant Difference test were used to further compare the differences at each time-point. SPSS 17.0 software (SPSS, Inc., Chicago, IL, USA) was used for all statistical analyses. $\mathrm{P}<0.05$ was considered to indicate a statistically significant difference between values.

\section{Results}

EA treatment improves the functional recovery of rats following stroke. In order to examine the effects of EA treatment on the functional recovery of MCAO rats, the mNSS was used to assess neurological deficits. The mNSS of the rats in the $S$ group were 0 at all time-points. As shown in Fig. 1A, the rats in the EA group had a significantly lower mNSS compared with that in the $\mathrm{M}$ group at $14(5.33 \pm 1.22$

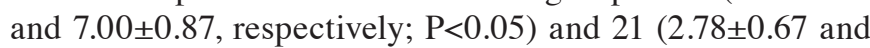
$4.78 \pm 0.67$, respectively; $\mathrm{P}<0.05$ ) days of treatment. Compared with those in the $\mathrm{M}$ group, rats in EA group only had significant improvement in the motor test score $(\mathrm{P}<0.05$; Fig. 1B) but not in scores of other tests at 14 and 21 days of treatment. The cognitive recovery of MCAO rats was further assessed using the Morris water maze test. As shown in Fig. 1C, rats in the EA group had significantly decreased escape latency compared with those in the $M$ group at 14 (90.63 \pm 2.62 and $98.13 \pm 2.90$, respectively; $\mathrm{P}<0.05)$ and $21(84.63 \pm 1.92$ and $94.13 \pm 1.46$, respectively; $\mathrm{P}<0.05)$ days of treatment. These results suggested that EA treatment effectively improved the neurological dysfunction of cerebral ischemia in rats, promoting nerve functional recovery.

EA treatment promotes the proliferation and differentiation of NSCs following stroke. BrdU/GFAP and BrdU/NeuN 
Table I. Modified Neurological Severity Score tests and points.

Test

Motor

Raising rat by tail 3

Flexion of forelimb

Flexion of hindlimb

Head moved $>10^{\circ}$ to vertical axis within $30 \mathrm{sec}$

Placing rat on floor (normal $=0$; maximum $=3$ )

Normal walk

Inability to walk straight

Circling toward paretic side

Falls down to paretic side

Sensory

Placing test (visual and tactile test)

Proprioceptive test (deep sensation, pushing paw against table edge to stimulate limb muscles)

Beam balance (normal=0; maximum $=6$ )

Balances with steady posture

Grasps side of beam

Hugs beam and 1 limb falls down from beam

Hugs beam and 2 limbs fall down from beam, or spins on beam ( $>60 \mathrm{sec})$

Attempts to balance on beam but falls off ( $>40 \mathrm{sec})$

Attempts to balance on beam but falls off $(>20 \mathrm{sec})$

Falls off; no attempt to balance or hang on to beam $(<20 \mathrm{sec})$

Reflex absence and abnormal movements

Pinna reflex (head shake when auditory meatus is touched)

Corneal reflex (eye blink when cornea is lightly touched with cotton)

Startle reflex (motor response to a brief noise from snapping a clipboard paper)

Seizures, myoclonus, myodystony

Maximum points

One point was awarded for inability to perform the task or for lack of reflex: 13-18, severe injury; 7-12, moderate injury; 1-6, mild injury.

A

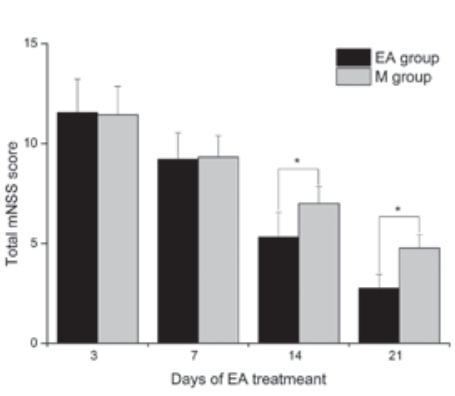

B

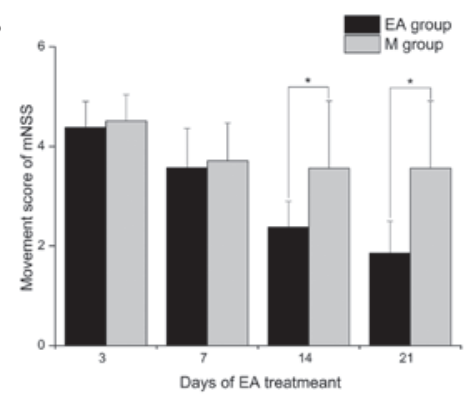

C

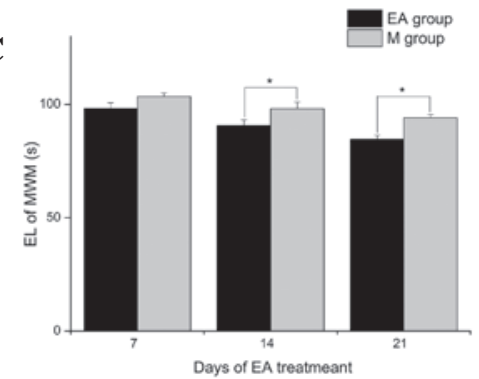

Figure 1. EA treatment significantly improves the recovery of neurological function in rats following stroke. (A) Neurological deficits and (B) motor deficits assessed by mNSS. (C) Recognitive deficits assessed by MWM. Values are presented as the mean \pm standard deviation. " $\mathrm{P}<0.05$ vs. M group. EA, eletroacupuncture; M, middle carotid artery occlusion control; mNSS, modified Neurological Severity Score; MWM, Morris water maze; EL, escape latency. 

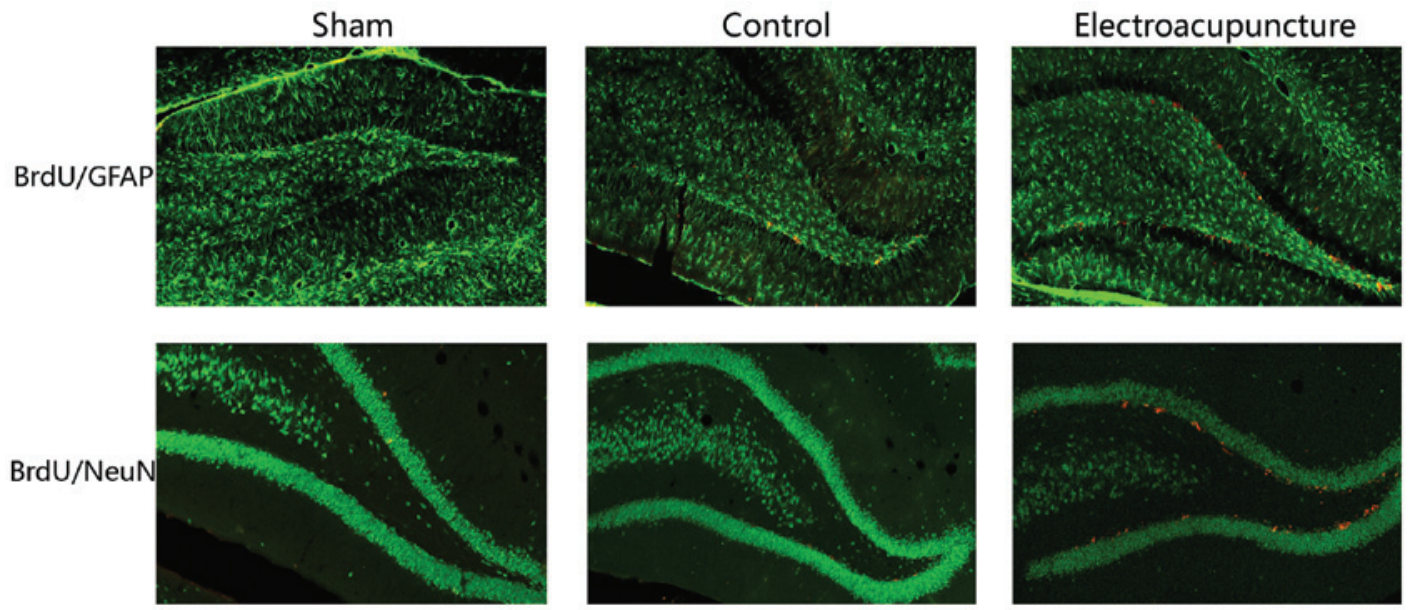

Figure 2. Representative images of $\mathrm{BrdU}^{+} / \mathrm{GFAP}^{+}$and $\mathrm{BrdU}^{+} / \mathrm{NeuN}^{+}$cells in the dentate gyrus area of rats on day 14 of electroacupuncture treatment, following immunofluorescence labeling. Green, BrdU; red, GFAP or NeuN. BrdU, bromodeoxyuridine; GFAP, glial fibrillary acidic protein; NeuN, neuronal nuclei.
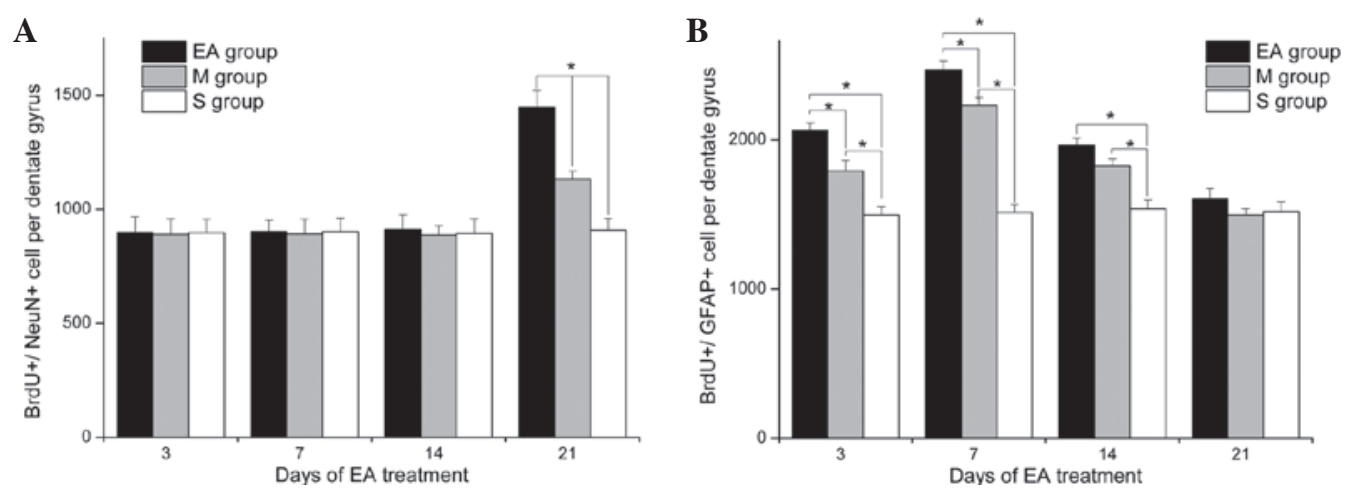

Figure 3. EA treatment significantly increases the number of $\mathrm{BrdU}^{+} / \mathrm{NeuN}^{+}$cells in the dentate gyrus of rats following stroke. Quantitative analyses of (A) $\mathrm{BrdU}^{+} / \mathrm{NeuN}^{+}$and (B) $\mathrm{BrdU}^{+} / \mathrm{GFAP}^{+}$cells on days 3, 7, 14 and 21 of EA treatment. Values are presented as the mean \pm standard deviation. ${ }^{*} \mathrm{P}<0.05$ between the indicated groups. BrdU, bromodeoxyuridine; GFAP, glial fibrillary acidic protein; NeuN, neuronal nuclei; EA, eletroacupuncture; M, middle carotid artery occlusion control; S, sham-operated.
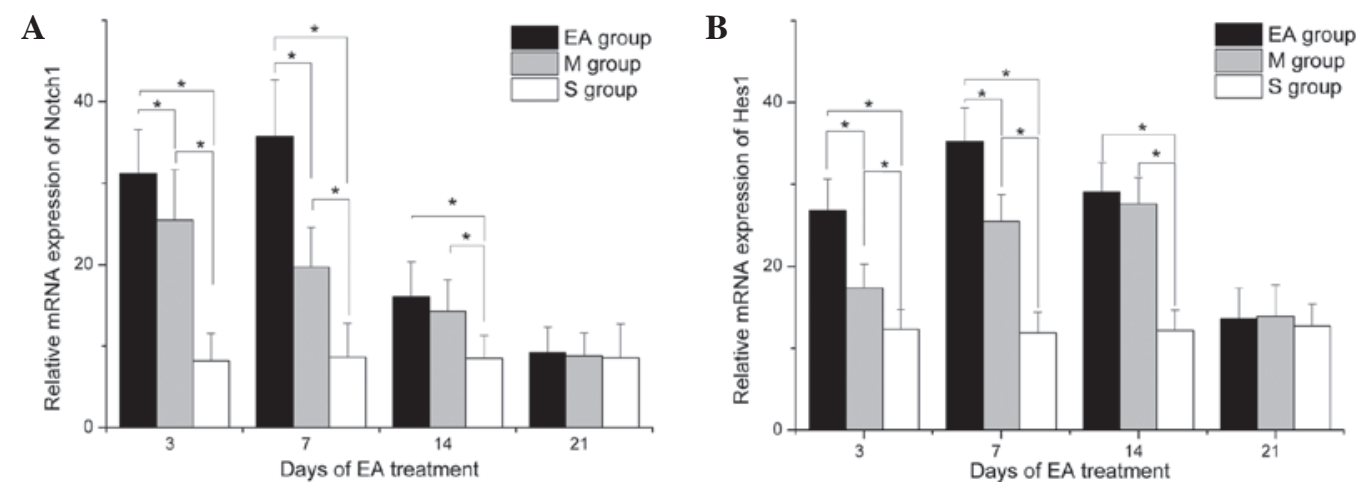

Figure 4. EA treatment significantly enhances the expression levels of Notch1 and Hes1 in the dentate gyrus area of rats following stroke. The mRNA expression levels of (A) Notch1 and (B) Hes were assessed by reverse transcription quantitative polymerase chain reaction at days 3, 7, 14 and 21 of EA treatment. Values are presented as the mean \pm standard deviation. ${ }^{*} \mathrm{P}<0.05$ between the indicated groups. EA, eletroacupuncture; $\mathrm{M}$, middle carotid artery occlusion control; S, sham-operated.

double-labeling immunofluorescence was performed to evaluate the proliferation and differentiation of NSCs following MCAO in each group. As shown in Figs. 2 and 3, MCAO rats had significantly higher frequencies of $\mathrm{BrdU}^{+} / \mathrm{GFAP}^{+}$and $\mathrm{BrdU}^{+} / \mathrm{NeuN}^{+}$cells in the DG area compared with those in the $\mathrm{S}$ group $(\mathrm{P}<0.05)$, indicating that the proliferation and differentiation of NSCs was stimulated by ischemic stress. In addition, EA treatment significantly elevated the frequencies of $\mathrm{BrdU}^{+} / \mathrm{GFAP}^{+}$cells at 3, 7 and 14 days of treatment compared with the control treatment $(\mathrm{P}<0.05)$, indicating the enhanced effects of EA treatment on the differentiation of NSCs into glia cells. Furthermore, EA treatment enhanced the neuronal 
differentiation of NSCs, as indicated by increased frequencies of $\mathrm{BrdU}^{+} / \mathrm{NeuN}^{+}$cells in the EA group, compared with that of the $\mathrm{M}$ group at 21 days of treatment $(\mathrm{P}<0.05)$.

EA treatment enhances the expression of Hotchl and Hes 1 following stroke. In order to examine the underlying molecular mechanisms by which EA treatment promoted the proliferation and differentiation of NSCs, the expression of Notch1 and its target molecule, Hes1, were analyzed by RT-qPCR. The results revealed that MCAO stress significantly stimulated the expression of Notch1 and Hes1 in the DG area compared with that of the $\mathrm{S}$ group at 3,7 and 14 days of treatment $(\mathrm{P}<0.05$; Fig. 4$)$.

\section{Discussion}

In the present study, the effects of EA treatment on the recovery of neurological factions and on the proliferation and differentiation of NSCs following stroke were investigated. The results revealed that EA treatment significantly improved the recovery of neural function in rats with MCAO. In addition, to the best of our knowledge, the present study was the first to demonstrate that EA treatment significantly promoted the proliferation and differentiation of NSCs into glial cells and neurons, possibly through the activation of Notch1 signaling pathways.

Previous studies have demonstrated that acupuncture or EA treatment promoted the recovery of neurological functions following stroke (22). In accordance with these findings, the present study confirmed that EA treatment significantly improved the mNSS and the Morris water maze performance of rats at 14 and 21 days of treatment following MCAO. It has been reported that EA treatment exerted its neuroprotective effects through a series of mechanisms, including reducing the apoptosis of neural cells and inhibiting the inflammatory responses (22). In addition, EA treatment has been found to enhance neurogenesis via the upregulation of neurotropic factors, including BDNF (30). However, the impact of EA treatment on the proliferation and differentiation of NSCs remains to be elucidated.

NSCs in the DG area of hippocampus are important in neurogenesis (31). In normal physiological conditions, these cells gradually generate neurons to renew the granular layer of the cortex throughout adult life. Pathological stress, including ischemic insult, markedly stimulates the neurogenesis of NSCs in the DG area. These characteristics suggest NSCs may offer a promising therapeutic strategy in stroke treatment. However, difficulties in the availability of abundant sources of NSCs and issues in legislation and ethics impede the clinical application of NSC transplantation treatment for stroke (32). Therefore, enhancing the expansion and differentiation of endogenous NSCs offers a promising alternative strategy for NSC treatment. In the present study, EA treatment was found to significantly promote the proliferation and differentiation of NSCs into glial cells and neurons, as indicated by increased $\mathrm{BrdU}^{+} / \mathrm{GFAP}^{+}$and $\mathrm{BrdU}^{+} / \mathrm{NeuN}^{+}$cell frequencies, respectively. However, in line with previous studies, the proliferation and differentiation of NSCs in the DG area in the M or EA groups only lasted for 3 weeks. These data highlight the requirement for the development of novel strategies to extend the duration and intensity of neurogenesis following stroke.
Notch1 is an essential regulator of NSC maintenance and self-renewal during development. In addition, components of the Notch signaling pathway are expressed in neuroproliferative regions of the postnatal brain (33). Previous studies have reported that Notch signaling may augment the expansion and differentiation of adult NSCs following stroke. Activated Notch1 (NICD) and its downstream transcriptional targets, including Hes1, have been identified in SVZ cells following ischemic injury (34-36). The expression levels of NICD and Hes1 are significantly upregulated in the SVZ at $4 \mathrm{~h}$ following focal cerebral ischemia. In addition, Notch1 signaling activation enhances SVZ cell proliferation, whereas inhibition of Notch1 signaling results in the reduced proliferation of cells in the SVZ. It has also been reported that, during differentiation, the expression levels of Notch and Hes1 are downregulated in neural progenitor cells following ischemia, which is associated with a significant increase in the size of the neuronal population. This suggests that Notch signaling is involved in mediating adult SVZ neural progenitor cell proliferation and differentiation following stroke (37). In line with these findings, the present study confirmed that stroke significantly increased the expression levels of Notch1 and Hes1. In addition, EA treatment significantly enhanced the expression levels of Notch1 and Hes1 stimulated by ischemia insult, which may, at least in part, account for the higher frequencies of $\mathrm{BrdU}^{+} / \mathrm{GFAP}^{+}$and $\mathrm{BrdU}^{+} / \mathrm{NeuN}^{+}$cells in the $\mathrm{DG}$ area of rats in the EA group.

In conclusion, to the best of our knowledge, the present study was the first to demonstrate that EA treatment promoted the neurogenesis of NSCs through upregulation of the expression of Notch1 following stroke. These findings contribute to the current understanding of the mechanisms by which acupuncture promotes the recovery of neurological functions following stroke and may provide support for novel integrative therapeutic strategies for stroke treatment.

\section{Acknowledgements}

The present study was supported by the National Natural Science Foundation of China (no. 81171863).

\section{References}

1. Kavanagh S, Knapp M and Patel A: Costs and disability among stroke patients. J Public Health Med 21: 385-394, 1999.

2. De Keyser J, Sulter G and Luiten PG: Clinical trials with neuroprotective drugs in acute ischaemic stroke: are we doing the right thing? Trends Neurosci 22: 535-540, 1999.

3. Liebeskind DS and Kasner SE: Neuroprotection for ischaemic stroke: an unattainable goal? CNS Drugs 15: 165-174, 2001.

4. Gage FH and Temple S: Neural stem cells: generating and regenerating the brain. Neuron 80: 588-601, 2013.

5. Zhao C, Deng W and Gage FH: Mechanisms and functional implications of adult neurogenesis. Cell 132: 645-660, 2008.

6. Reynolds BA and Weiss S: Generation of neurons and astrocytes from isolated cells of the adult mammalian central nervous system. Science 255: 1707-1710, 1992.

7. Weiss S, Reynolds BA, Vescovi AL, Morshead C, Craig CG and van der Kooy D: Is there a neural stem cell in the mammalian forebrain? Trends Neurosci 19: 387-393, 1996.

8. Reynolds BA and Weiss S: Clonal and population analyses demonstrate that an EGF-responsive mammalian embryonic CNS precursor is a stem cell. Dev Biol 175: 1-13, 1996.

9. McKay R: Stem cells in the central nervous system. Science 276: 66-71, 1997. 
10. Liu J, Solway K, Messing RO and Sharp FR: Increased neurogenesis in the dentate gyrus after transient global ischemia in gerbils. J Neurosci 18: 7768-7778, 1998

11. Jin K,Minami M,Lan JQ, et al: Neurogenesis in dentate subgranular zone and rostral subventricular zone after focal cerebral ischemia in the rat. Proc Natl Acad Sci USA 98: 4710-4715, 2001.

12. Arvidsson A, Collin T, Kirik D, Kokaia Z and Lindvall O Neuronal replacement from endogenous precursors in the adult brain after stroke. Nat Med 8: 963-970, 2002

13. Takasawa K, Kitagawa K, Yagita Y, et al: Increased proliferation of neural progenitor cells but reduced survival of newborn cells in the contralateral hippocampus after focal cerebral ischemia in rats. J Cereb Blood Flow Metab 22: 299-307, 2002.

14. Ming GL and Song H: Adult neurogenesis in the mammalian brain: significant answers and significant questions. Neuron 70: 687-702, 2011.

15. Lie DC, Song H, Colamarino SA, Ming GL and Gage FH: Neurogenesis in the adult brain: new strategies for central nervous system diseases. Annu Rev Pharmacol Toxicol 44: 399-421, 2004

16. Ceniceros S and Brown GR: Acupuncture: a review of its history, theories and indications. South Med J 91: 1121-1125, 1998.

17. Yang R, Huang ZN and Cheng JS: Anticonvulsion effect of acupuncture might be related to the decrease of neuronal and inducible nitric oxide synthases. Acupunct Electrother Res 24: 161-167, 1999.

18. Junhua Z, Menniti-Ippolito F, Xiumei G, et al: Complex traditional Chinese medicine for poststroke motor dysfunction: a systematic review. Stroke 40: 2797-2804, 2009.

19. Ulett GA, Han S and Han JS: Electroacupuncture: mechanisms and clinical application. Biol Psychiatry 44: 129-138, 1998.

20. Tong RK, Ng MF and Li LS: Effectiveness of gait training using an electromechanical gait trainer, with and without functional electric stimulation, in subacute stroke: a randomized controlled trial. Arch Phys Med Rehabil 87: 1298-1304, 2006.

21. Yan T, Hui-Chan CW and Li LS: Functional electrical stimulation improves motor recovery of the lower extremity and walking ability of subjects with first acute stroke: a randomized placebo-controlled trial. Stroke 36: 80-85, 2005.

22. Ho TJ, Chan TM, Ho LI, Lai CY, Lin CH, Macdonald I, Harn HJ, Lin JG, Lin SZ and Chen YH: The possible role of stem cells in acupuncture treatment for neurodegenerative diseases: a literature review of basic studies. Cell Transplant 23: 559-566, 2014.

23. Ke Z, Yip SP, Li L, Zheng XX and Tong KY: The effects of voluntary, involuntary and forced exercises on brain-derived neurotrophic factor and motor function recovery: a rat brain ischemia model. PLoS One 6: e16643, 2011.

24. Xi GM, Wang HQ, He GH, Huang CF and Wei GY: Evaluation of murine models of permanent focal cerebral ischemia. Chin Med J (Engl) 117: 389-394, 2004.
25. Yang Y, Li Q, Miyashita H, Howlett W, Siddiqui M and Shuaib A: Usefulness of postischemic thrombolysis with or without neuroprotection in a focal embolic model of cerebral ischemia. J Neurosurg 92: 841-847, 2000.

26. Yang Y,Li Q and Shuaib A: Neuroprotection by 2-h postischemia administration of two free radical scavengers, alpha-phenyl-n-te rt-butyl-nitrone (PBN) and N-tert-butyl-(2-sulfophenyl)-nitrone (S-PBN), in rats subjected to focal embolic cerebral ischemia. Exp Neurol 163: 39-45, 2000.

27. Chen J, Sanberg PR, Li Y, et al: Intravenous administration of human umbilical cord blood reduces behavioral deficits after stroke in rats. Stroke 32: 2682-2688, 2001.

28. Mohapel P, Mundt-Petersen K, Brundin P and Frielingsdorf H: Working memory training decreases hippocampal neurogenesis. Neuroscience 142: 609-613, 2006.

29. Chen Y, Pan K, Li S, Xia J, Wang W, Chen J, Zhao J, Lü L, Wang D, Pan Q, et al: Decreased expression of V-set and immunoglobulin domain containing 1 (VSIG1) is associated with poor prognosis in primary gastric cancer. J Surg Oncol 106: 286-293, 2012.

30. Kim MW, Chung YC, Jung HC, et al: Electroacupuncture enhances motor recovery performance with brain-derived neurotrophic factor expression in rats with cerebral infarction. Acupunct Med 30: 222-226, 2012.

31. Gu Y, Janoschka S and Ge S: Neurogenesis and hippocampal plasticity in adult brain. Curr Top Behav Neurosci 15: 31-48, 2013.

32. Grisolía JS: CNS stem cell transplantation: clinical and ethical perspectives. Brain Res Bull 57: 823-826, 2002.

33. Piccin D, Yu F and Morshead CM: Notch signaling imparts and preserves neural stem characteristics in the adult brain. Stem Cells Dev 22: 1541-1550, 2013.

34. Pujia F, Serrao M, Brienza M, et al: Effects of a selective serotonin reuptake inhibitor escitalopram on the cutaneous silent period: A randomized controlled study in healthy volunteers. Neurosci Lett 566: 17-20, 2014.

35. Guo YJ, Zhang ZJ, Wang SH, Sui YX and Sun Y: Notch1 signaling, hippocampal neurogenesis and behavioral responses to chronic unpredicted mild stress in adult ischemic rats. Prog Neuropsychopharmacol Biol Psychiatry 33: 688-694, 2009.

36. Chen J, Zacharek A, Li A, et al: Atorvastatin promotes presenilin-1 expression and Notch1 activity and increases neural progenitor cell proliferation after stroke. Stroke 39: 220-226, 2008.

37. Wang L, Chopp M, Zhang RL, et al: The Notch pathway mediates expansion of a progenitor pool and neuronal differentiation in adult neural progenitor cells after stroke. Neuroscience 158 : $1356-1363,2009$ 